\title{
Recycling of plastic waste into fuels, a review
}

\section{Hayelom Dargo Beyene}

Adigrat University, Department of Chemistry, Adigrat, Ethiopia, P.Box .50

\section{Email address:}

hayeda21@gmail.com

\section{To cite this article:}

Hayelom Dargo Beyene. Recycling of Plastic Waste into Fuels, a Review. International Journal of Science, Technology and Society. Vol. 2, No. 6, 2014, pp. 190-195. doi: 10.11648/j.ijsts.20140206.15

\begin{abstract}
Plastics have become common materials of our everyday lives, and many of their properties, such as durability, versatility and lightweight, can be a significant factor in achieving sustainable development. A common problem with recycling plastics is that plastics are often made up of more than one kind of polymer or there may be some sort of fiber added to the plastic (a composite) to give added strength. This can make recovery difficult. Rejected or waste material usually has good characteristics for recycling and will be clean. Although the quantity of material available is sometimes small, the quantities tend to be growing as consumption, and therefore production, increases. Recycling is a method preventing accumulation the excessive waste quantity through their second processing and utilization in the production process of new materials. The aim of this review was focused on the introducing of the technologies (methods) used to convert waste plastic to fuels and to call researchers on production of fuels from waste plastic and other related materials.
\end{abstract}

Keywords: Fuels, Plastic, Recycling, Polymer, Recycling Methods

\section{Introduction}

As a brief introduction to plastics, it can be said that plastics are synthetic organic materials produced by polymerization. They are typically of high molecular mass, and may contain other substances besides polymers to improve performance and/or reduce costs. These polymers can be molded or extruded into desired shapes. There are two main types of plastics: thermoplastics and thermosetting polymers. Thermoplastics can repeatedly soften and melt if enough heat is applied and hardened on cooling, so that they can be made into new plastics products. Examples are polyethylene, polystyrene and polyvinyl chloride, among others. Thermosets or thermo settings can melt and take shape only once. They are not suitable for repeated heat treatments; therefore after they have solidified, they stay solid. Examples are phenol formaldehyde and urea formaldehyde.

Plastics have become common materials of our everyday lives, and many of their properties, such as durability, versatility and light-weight, can be a significant factor in achieving sustainable development. However, plastic applications also contribute to the growing amounts of solid waste generated, as plastic products are often used only once before disposal. The disposal problem is not simply technical, but it also has social, economic and even political aspects. This is the reason why several different methods have been explored and applied for solving the problems associated with polymer waste handling and disposal [1].

\subsection{Common Plastics for Recycling}

Not all plastics are recyclable. There are 4 types of plastic which are commonly recycled:

- Polyethylene (PE) - both high density and low-density polyethylene.

- Polypropylene (PP)

- Polystyrene (PS)

- Polyvinyl chloride (PVC)

A common problem with recycling plastics is that plastics are often made up of more than one kind of polymer or there may be some sort of fiber added to the plastic (a composite) to give added strength. This can make recovery difficult.

\section{Identification of different types of plastics}

There are several simple tests that can be used to distinguish between the common types of polymers so that they may be separated for processing. 
Table 1. Identification of different types of plastics

\begin{tabular}{lllll}
\hline Test & PE & PP & PS & PVC \\
\hline Water & Floats & Floats & Sinks & Sinks \\
Burning & Blue flame with yellow & Yellow flame with blue base. & Yellow, sooty flame - & Yellow, sooty, Smoke. Does not \\
& tip, elts and drips. & Like candle wax- less strong & Sweet & Hydrochloric acid \\
Smell after burning & Like candle wax & than PE & No & No \\
\hline Scratch & Yes & No & Some is removed \\
\hline
\end{tabular}

Waste plastics are one of the most promising resources for fuel production because of its high heat of combustion and due to the increasing availability in local communities. Unlike paper and wood, plastics do not absorb much moisture and the water content of plastics is far lower than the water content of biomass such as crops and kitchen wastes. The conversion methods of waste plastics into fuel depend on the types of plastics to be targeted and the properties of other wastes that might be used in the process. Additionally the effective conversion requires appropriate technologies to be selected according to local economic, environmental, social and technical characteristics [2].

In general, the conversion of waste plastic into fuel requires feedstocks which are non-hazardous and combustible. In particular each type of waste plastic conversion method has its own suitable feedstock. The composition of the plastics used as feedstock may be very different and some plastic articles might contain undesirable substances (e.g. additives such as flame-retardants containing bromine and antimony compounds or plastics containing nitrogen, halogens, sulfur or any other hazardous substances) which pose potential risks to humans and to the environment. The types of plastics and their composition will condition the conversion process and will determine the pretreatment requirements, the combustion temperature for the conversion and therefore the energy consumption required, the fuel quality output, the flue gas composition (e.g. formation of hazardous flue gases such as $\mathrm{NOx}$ and $\mathrm{HCl}$ ), the fly ash and bottom ash composition, and the potential of chemical corrosion of the equipment, Therefore the major quality concerns when converting waste plastics into fuel resources are as follows:

1) Smooth feeding to conversion equipment: Prior to their conversion into fuel resources, waste plastics are subject to various methods of pretreatment to facilitate the smooth and efficient treatment during the subsequent conversion process. Depending on their structures (e.g. rigid, films, sheets or expanded (foamed) material) the pretreatment equipment used for each type of plastic (crushing or shredding) is often different.

2) Effective conversion into fuel products: In solid fuel production, thermoplastics act as binders which form pellets or briquettes by melting and adhering to other non-melting substances such as paper, wood and thermosetting plastics. Although wooden materials are formed into pellets using a pelletizer, mixing plastics with wood or paper complicates the pellet preparation process. Suitable heating is required to produce pellets from thermoplastics another combustible waste. In liquid fuel production, thermoplastics containing liquid hydrocarbon can be used as feedstock. The type of plastic being used determines the processing rate as well as the product yield. Contamination by undesirable substances and the presence of moisture increases energy consumption and promotes the formation of byproducts in the fuel production process.

3) Well-controlled combustion and clean flue gas in fuel user facilities: It is important to match the fuel type and its quality to the burner in order to improve heat recovery efficiency. Contamination by nitrogen, chlorine, and inorganic species, for instance, can affect the flue gas composition and the amount of ash produced. When using fuel prepared from waste plastics, it must be assured that the flue gas composition complies with local air pollution regulations. In the same way, ash quality must also be in compliance with local regulations when disposed at the landfill. If there aren't any relevant regulations, both the producers and consumers of the recycled fuel should control the fuel quality and the emissions at combustion in order to minimize their environmental impact.

Table 2 classifies various plastics according to the types of fuel they can produce. It can be observed that thermoplastics consisting of carbon and hydrogen are the most important feedstock for fuel production either in solid or liquid form. As shown in Table 2. PE, PP and PS thermoplastics are preferable as feedstock in the production of liquid hydrocarbons. The addition of thermosetting plastics, wood, and paper to the feedstock leads to the formation of carbonous substances and lowers the rate and yield of liquid products.

Table 2. Polymer as feedstock for fuel production

\begin{tabular}{|c|c|c|}
\hline Types of polymer & Descriptions & Examples \\
\hline Polymers consisting of carbon and hydrogen & $\begin{array}{l}\text { Typical feedstock for fuel production due to high heat } \\
\text { value and clean exhaust gas. }\end{array}$ & $\begin{array}{l}\text { Polyethylene, polypropylene, polystyrene. } \\
\text { Thermoplastics melt to form solid fuel mixed } \\
\text { with other combustible wastes and Decompose to } \\
\text { produce liquid fuel. }\end{array}$ \\
\hline Polymers containing oxygen & Lower heat value than above plastics & $\begin{array}{l}\text { PET, phenolicresin, polyvinyl alcohol, } \\
\text { polyoxymethylene }\end{array}$ \\
\hline
\end{tabular}




\begin{tabular}{lll}
\hline Types of polymer & Descriptions & Examples \\
\hline $\begin{array}{l}\text { PET, phenolic resin, polyvinyl alcohol, } \\
\text { polyoxymethylene, Polymers containing, } \\
\text { nitrogen or sulfur }\end{array}$ & $\begin{array}{l}\text { Fuel from this type of plastic is a source of hazardous } \\
\text { components such as NOx or Sox in flue gas. Flue gas } \\
\text { cleaning is required to avoid emission of hazardous } \\
\text { components in exhaust gas. }\end{array}$ & $\begin{array}{l}\text { Nitrogen,polyamide, polyurethane,sulfur: } \\
\text { polyphenylene sulfide }\end{array}$ \\
$\begin{array}{l}\text { Polymers containing } \\
\text { halogens of chlorine, } \\
\text { bromine and fluorine }\end{array}$ & $\begin{array}{l}\text { Source of hazardous and corrosive flue gas upon } \\
\text { thermal treatment and combustion. }\end{array}$ & $\begin{array}{l}\text { Polyvinylchloride, polyvinylidene, chloride, } \\
\text { bromine-containing flame retardants and } \\
\text { fluorocarbon polymers }\end{array}$ \\
\hline
\end{tabular}

Table 3. Product types of some plastics pyrolysis

\begin{tabular}{|c|c|c|}
\hline Main products & Type of plastics & As a feedstock of liquid fuel \\
\hline Liquid hydrocarbons & $\begin{array}{l}\text { Polyethylene (PE) Polypropylene (PP) Polystyrene (PS) } \\
\text { Polymethyl metacrylate (PMMA) }\end{array}$ & Allowed. Allowed. Allowed. Allowed \\
\hline Liquid hydrocarbons & Acrylonitrile-Butadiene-Styrene copolymer (ABS) & $\begin{array}{l}\text { Allowed. But not suitable. Nitrogen-containing fuel is } \\
\text { obtained. Special attention required to cyanide in oil. }\end{array}$ \\
\hline No hydrocarbons suitable for fuel & Polyvinyl alcohol (PVA) Polyoxymethylene (POM & $\begin{array}{l}\text { Not suitable. Formation of water and alcohol. Not } \\
\text { suitable. Formation of Formaldehyde. }\end{array}$ \\
\hline Solid products & Polyethylene terephthalate (PET) & $\begin{array}{l}\text { Not suitable. Formation of Terephthalic acid and } \\
\text { benzoic acid. }\end{array}$ \\
\hline Carbon & Poly & Not suitable. Not suitable. \\
\hline Carbonous products & Polyvinyl chloride (PVC) Polyvinylidene chloride (PVDC) & Not allowed. Not allowed. \\
\hline
\end{tabular}

\subsection{Sources of Plastic Waste}

Industrial waste (or primary waste) can often be obtained from the large plastics processing, manufacturing and packaging industries. Rejected or waste material usually has good characteristics for recycling and will be clean. Although the quantity of material available is sometimes small, the quantities tend to be growing as consumption, and therefore production, increases [3].Commercial waste is often available from workshops, craftsmen shops, supermarkets and wholesalers. A lot of the plastics available from these sources will be PE, often contaminated. Agricultural waste can be obtained from farms and nursery gardens form of packaging (plastic containers or sheets) or construction materials (irrigation or hosepipes).

\section{Recycling Methods}

\subsection{Chemical Depolymerisation}

Chemical depolymerisation, or chemolysis, involves the reaction of the used polymer with chemical reagents for the production of its starting monomers. Different processes have been developed which are categorized depending on the chemical agents employed, the most common being glycolysis, methanolysis, hydrolysis and ammonolysis [4].

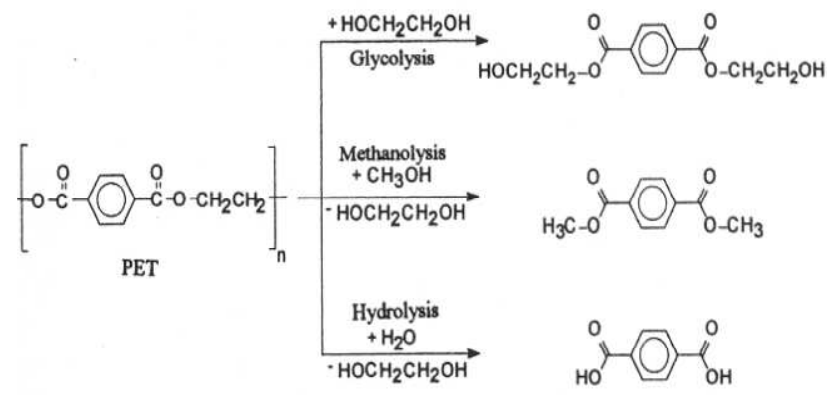

Figure 1. Shows Chemical Depolymerisation of PET

\subsection{Gasification}

Gasification involves the partial oxidation of organic matter at high temperatures (typically between $1200-1500^{\circ} \mathrm{C}$ ) under mildly oxidizing conditions (usually steam, carbon dioxide or Sub-stoichiometric oxygen) for the production of synthesis gas (syngas). This gas, consisting primarily of carbon monoxide and hydrogen, has application in the synthesis of chemicals like methanol and ammonia, and can be used to produce synthetic diesel or may be combusted directly as a fuel [5].

$$
\begin{aligned}
& \text { - Raw material decomposition } \\
& C_{x} H_{y} \Leftrightarrow x C+\frac{y}{2} H_{2} \\
& \text { - Reactions with oxygen } \\
& \mathrm{C}+\frac{1}{2} \mathrm{O}_{2} \Leftrightarrow \mathrm{CO} \\
& \mathrm{CO}+\frac{1}{2} \mathrm{O}_{2} \Leftrightarrow \mathrm{CO}_{2} \\
& \mathrm{H}_{2}+\frac{1}{2} \mathrm{O}_{2} \Leftrightarrow \mathrm{H}_{2} \mathrm{O} \\
& \mathrm{C}_{\mathrm{x}} \mathrm{H}_{\mathrm{y}}+\left(\mathrm{x}+\frac{\mathrm{y}}{4}\right) \mathrm{O}_{z} \Leftrightarrow \mathrm{xCO}_{2}+\frac{\mathrm{y}}{2} \mathrm{H}_{2} \mathrm{O} \\
& \mathrm{C}_{\mathrm{x}} \mathrm{H}_{\mathrm{y}}+\left(\frac{\mathrm{x}}{2}+\frac{\mathrm{y}}{4}\right) \mathrm{O}_{2} \Leftrightarrow \mathrm{xCO}+\frac{\mathrm{y}}{2} \mathrm{H}_{2} \mathrm{O} \\
& \mathrm{C}_{\mathrm{x}} \mathrm{H}_{\mathrm{y}}+\mathrm{xH}_{2} \mathrm{O} \Leftrightarrow \mathrm{xCO}+\left(\frac{\mathrm{y}}{2}+\mathrm{x}\right) \mathrm{H}_{2} \\
& \mathrm{C}+\mathrm{CO}_{2} \Leftrightarrow 2 \mathrm{CO} \\
& \mathrm{C}_{\mathrm{X}} \mathrm{H}_{\mathrm{y}}+\mathrm{x} \mathrm{CO}_{2} \Leftrightarrow 2 \mathrm{xCO}+\frac{\mathrm{y}}{2} \mathrm{H}_{2} \\
& \mathrm{CO}+3 \mathrm{H}_{2} \Leftrightarrow \mathrm{CH}_{4}+\mathrm{H}_{2} \mathrm{O} \\
& \mathrm{CO}_{2}+4 \mathrm{H}_{2} \Leftrightarrow \mathrm{CH}_{4}+2 \mathrm{H}_{2} \mathrm{O}
\end{aligned}
$$




\subsection{Thermal Cracking}

Thermal cracking, or pyrolysis, involves the degradation of the polymeric materials by heating in the absence of oxygen. The process is usually conducted at temperatures between $500-800^{\circ} \mathrm{C}$ and results in the formation of a carbonized char and a volatile fraction that may be separated into condensable hydrocarbon oil and a non-condensable high calorific value gas. The proportion of each fraction and their precise composition depends primarily on the nature of the plastic waste but also on process conditions [6].

\subsection{Catalytic Conversions}

Catalytic conversion of plastic wastes implies several advantages over conventional pyrolytic methods. The most evident relates to the lower degradation temperatures at which degradation reaction takes place, which results in lower energy consumptions and higher conversion rates.

\subsection{Hydrogenation}

Hydrogenation of plastics is a potential alternative for breaking down the polymer chain. Compared to treatments in the absence of hydrogen, hydrogenation leads to the formation of highly saturated products, avoiding the presence of olefins in the liquid fractions, which favours their use as fuels without further treatments. Moreover, hydrogenation promotes the removal of hetero atoms, such as chlorine $(\mathrm{Cl})$, nitrogen $(\mathrm{N})$ and sulphur $(\mathrm{S})$, in the form of volatile compounds. However, hydrogenation suffers several drawbacks, mainly due to the cost of hydrogen and the need to operate under high pressure.

\section{Converting Into Liquid Fuel}

Liquid fuel is defined as plastic-derived liquid hydrocarbons at a normal temperature and pressure. Only several types of thermoplastics undergo thermal decomposition to yield liquid hydrocarbons used as liquid fuel. PE, PP, and PS, are preferred for the feedstock of the production of liquid hydrocarbons. The addition of thermosetting plastics, wood, and paper to feedstock leads to the formation of carbonous substance. It lowers the rate and yields of liquid products.

Depending on the components of the waste plastic being used as feedstock for fuel production, the resulting liquid fuel may contain other contaminants such as amines, alcohols, waxy hydrocarbons and some inorganic substances. Contamination of nitrogen, sulfur and halogens gives flu gas pollution. Unexpected contamination and high water contents may lower the product yields and shorten the lifetime of a reactor for pyrolysis.Liquid fuel users require petroleum substitutes such as gasoline, diesel fuel and heavy oil. In these fuels, various additives are often mixed with the liquid hydrocarbons to improve the burner or the engine performance. The fuel properties such as viscosity and ash content should conform to the specifications of the fuel user's burners or engines.

\subsection{Production Method}

The production method for the conversion of plastics to liquid fuel is based on the pyrolysis of the plastics and the condensation of the resulting hydrocarbons. Pyrolysis refers to the thermal decomposition of the matter under an inert gas like nitrogen. For the production process of liquid fuel, the plastics that are suitable for the conversion are introduced into a reactor where they will decompose at 450 to $550{ }^{\circ} \mathrm{C}$. Depending on the pyrolysis conditions and the type of plastic used, carbonous matter gradually develops as a deposit on the inner surface of the reactor. After pyrolysis, this deposit should be removed from the reactor in order to maintain the heat conduction efficiency of the reactor [6].

The resulting oil (mixture of liquid hydrocarbons) is continuously distilled once the waste plastics inside the reactor are decomposed enough to evaporate upon reaching the reaction temperature. The evaporated oil is further cracked with a catalyst. The boiling point of the produced oil is controlled by the operation conditions of the reactor, the cracker and the condenser. In some cases, distillation equipment is installed to perform fractional distillation to meet the user's requirements.

After the resulting hydrocarbons are distilled from the reactor, some hydrocarbons with high boiling points such as diesel, kerosene and gasoline are condensed in a water-cooled condenser. The liquid hydrocarbons are then collected in a storage tank through a receiver tank. Gaseous hydrocarbons such as methane, ethane, propylene and butanes cannot be condensed and are therefore incinerated in a flare stack. This flare stack is required when the volume of the exhaust gas emitted from the reactor is expected to be large.

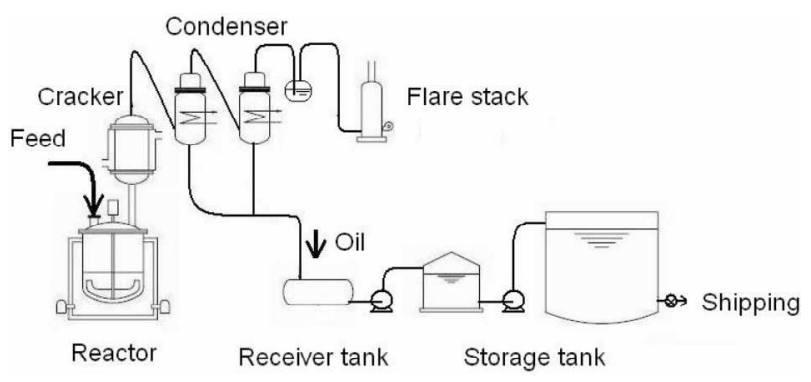

Figure 2. presents a schematic diagram of a liquid fuel production plant (Pyrolysis process).

There may be variations in the feeding methods used depending on the characteristics of the waste plastic. The easiest way is to simply introduce the waste plastics into the reactor without any pretreatment. Soft plastics such as films and bags are often treated with a shredder and a melter (hot melt extruder) in order to feed them into the reactor because otherwise they would occupy a large volume of the reactor.

There are also different types of reactors and heating equipment. Both kiln-type and Screw-type reactors have been proposed, while induction heating by electric power has been developed as an alternative to using a burner. Due to the formation of carbonous matter in the reactor, this acts as a heat insulator, in some tank reactors the stirrer is used to 
remove the carbonous matter rather than for stirring.

After the liquid product of the Pyrolysis is distilled, the carbonous matter is taken out either with a vacuum cleaner or in some cases reactors are equipped with a screw conveyor at the bottom of the tank reactor to remove the carbonous matter.

Operators should understand the relationship between the amount and composition of the waste plastics as well as the operating conditions. Energy consumption and plant costs relative to the plastic treatment capacity are the typical criteria for evaluating the plant performance.

Operating skill and safety considerations are important in this type of chemical conversion due to the highly flammable liquid fuels which are formed.

\section{Converting in to Gas Fuel}

The gaseous fuel refers to the flammable gas obtained from the thermal treatment of waste plastics. There are two types of gaseous fuel: Gaseous hydrocarbon: hydrocarbons that are in a gaseous state under normal temperature and pressure $\left(0{ }^{\circ} \mathrm{C}, 1 \mathrm{~atm}\right)$. Synthesis gas or syngas: mixture of hydrogen and carbon monoxide.

In the conversion of plastics to gaseous fuel, the waste plastics undergo thermal decomposition in a tank reactor, resulting in the formation of liquid fuel as the main product and gaseous fuel up to about $20 \mathrm{wt} \%$, as the minor product. Gaseous hydrocarbons become the main product after residing in the reactor for an extended time at a reaction temperature under controlled decomposition conditions and the use of a specific reactor. Under specific conditions, carbon and carbohydrates can be used as feedstocks for the production of gaseous fuel like methane and hydrogen.

\subsection{Production Method}

The gasification process includes a series of steps such as pretreatment, gasification, gas cleaning and storage. Polyethylene and polypropylene thermally decompose at temperatures up to about $700{ }^{\circ} \mathrm{C}$ and under a inert atmosphere to form a mixture of gaseous hydrocarbons, methane, ethane, ethylene, propane, propylene, and various isomers of butane and butane. On the other hand, most of the organic substances undergo gasification yielding synthesis gas [7].

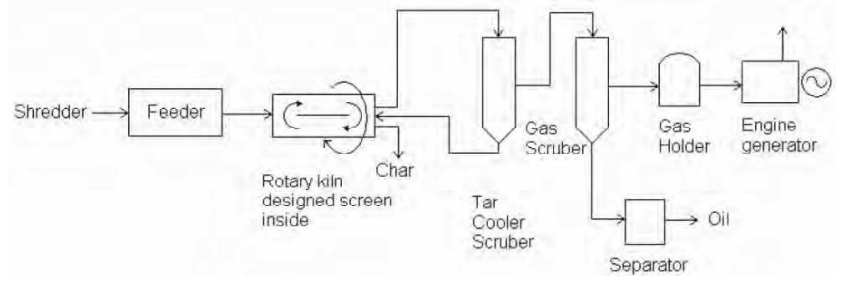

Figure 3. Schematic diagram of a production plant of plastics-derived gaseous fuel

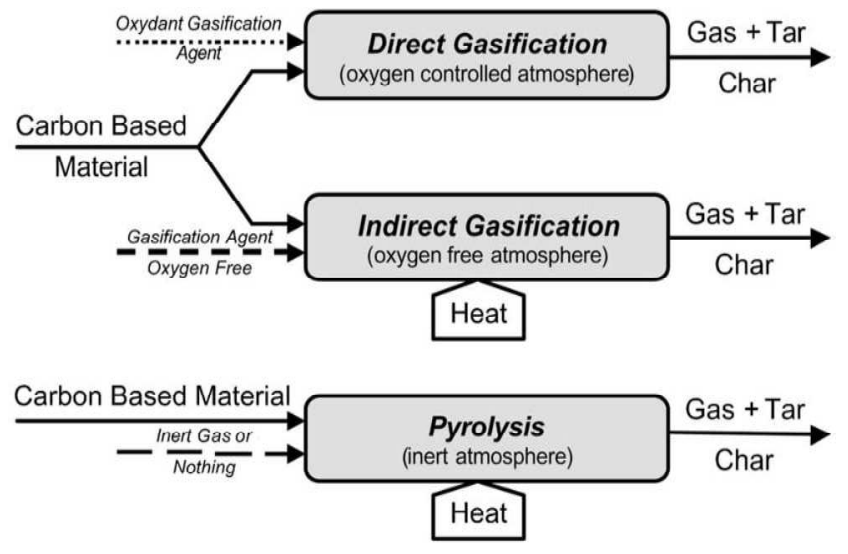

Figure 4. Gasification and pyrolysis processes

Gasification proceeds at elevated temperatures, higher than 800 and practically $1000^{\circ} \mathrm{C}$. Depending on the types of reactors and reaction conditions, carbonous matter and carbon dioxide are formed, and nitrogen from the air is contained in the product gas. Several manufacturers have proposed small-scale gasification systems. Careful cost analysis is important with respect to the amount of collected waste, the transportation distance and the commercial value of the resultant products such as electricity and gaseous fuel. In any case, this technology requires skillful operators and careful handling to avoid hydrogen explosion.

Table 4. List of various gasification methods

\begin{tabular}{|c|c|c|}
\hline Type of gasification & Conditions & Typical products \\
\hline Pyrolysis & $>700^{\circ} \mathrm{C}$ under inert atmosphere & $\begin{array}{l}\text { Gaseous hydrocarbons from aliphatic hydrocarbons including polyethylene and } \\
\text { Polypropylene. }\end{array}$ \\
\hline Partial oxidation & $>1000{ }^{\circ} \mathrm{C}$ under oxygen or air & $\begin{array}{l}\text { Carbon monoxide from carbon, hydrocarbons and Carbohydrates including wood. } \\
\text { Hydrogen also Forms from hydrocarbons and carbohydrates. }\end{array}$ \\
\hline Steam gasification & $>800^{\circ} \mathrm{C}$ under oxygen or air & Methane, carbon monoxide and hydrogen \\
\hline Hydro gasification & $\begin{array}{l}\text { Around } 500-600^{\circ} \mathrm{C} \text { under } \\
\text { hydrogen }\end{array}$ & Methane, carbon monoxide and water \\
\hline
\end{tabular}

Direct gasification occurs when an oxidant gasification agent is used to partially oxidize the feedstock [8]. The oxidation reactions supply the energy to keep the temperature of the process up. If the process does not occur with an oxidizing agent, it is called indirect gasification and needs an external energy source .Steam is the most commonly used indirect gasification agent, because it is easily produced and increases the hydrogen content of the combustible gas . Pyrolysis is an indirect gasification process with inert gases as the gasification agent.

As shown in Fig.3, resulting from the gasification process and varying with the temperature at which the process is 
carried out, the three major output fractions are

1. a combustible gas;

2. a liquid fraction (tars and oils); and

3. a char, consisting of almost pure carbon plus inert material originally present in the feedstock.

\section{Conclusions}

Recycling is a method preventing accumulation the excessive waste quantity through their second processing and utilization in the production process of new materials. Nowadays it is considered as a method developing the most spectrum of the environmental protection. Plastic wastes can recycle into valuable gas and liquid fuels by the through chemical recycling methods such as Hydrogenation, Chemical depolymerisation, Gasification, Thermal cracking, Catalytic conversions.

\section{References}

[1] Vogler, Jon, Small-scale recycling of plastics. Intermediate Technology Publications2005.
[2] Jefferson. H, Robert .D and Edward. K, Plastics recycling: challenges and opportunities, 2011, September 29.

[3] Moinuddin Sarker, Mohammad. M , Agricultural waste plastics conversion into high energy liquid hydrocarbon fuel by thermal degradation process, Journal of Petroleum Technology and Alternative Fuels,2011, Vol. 2(8), pp. 141145 .

[4] J. Aguado D.P.\& Serrano.G,European trends in the feedstock recycling of plastic wastes, global nest Journal, 2007, Vol. 9, No 1, pp 12-19.

[5] V. Belgiorno, G. De Feo, Energy from gasification of solid wastes, 2003, pp 1-15.

[6] Guang-Hua Zhang ,Jun-Feng Zhu, Prospect and current status of recycling waste plastics and technology for converting them into oil in China, 2006,pp 231-239.

[7] Jenni, Ylä-Mella, Recycling of polymers, Department of Process and Environmental Engineering, 2005,pp.1-9.

[8] S.M. Al-Salem ,P.Lettieri, Recycling and recovery routes of plastic solid waste (PSW), (2009),vol. 29,pp. 2625-2643 